\title{
Development of a mid-infrared nitrogen dioxide sensor based on Faraday rotation spectroscopy
}

\author{
Kun Liu ${ }^{1,2}$, Rafał Lewicki ${ }^{3}$, and Frank K. Tittel ${ }^{1, \bigotimes}$ \\ ${ }^{1}$ Department of Electrical and Computer Engineering, Rice University, Houston, Texas 77005, USA \\ ${ }^{2}$ Anhui Institute of Optics \& Fine Mechanics, Chinese Academy of Sciences, Hefei 230031, China \\ ${ }^{3}$ Intense, North Brunswick, NJ 08902

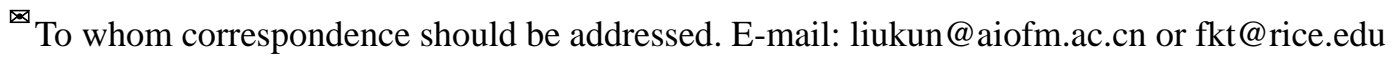

\begin{abstract}
A sensitive nitrogen dioxide $\left(\mathrm{NO}_{2}\right)$ sensor system based on Faraday Rotation Spectroscopy (FRS) was developed. The advantages of FRS include high detection sensitivity, zero background and free from the influence of diamagnetic species, such as humidity and carbon dioxide. A widely tunable external cavity quantum cascade laser (EC-QCL) was used as the excitation source. The tunable EC-QCL operates mode-hop free between $1600 \mathrm{~cm}^{-1}$ and $1650 \mathrm{~cm}^{-1}$ and allows targeting the optimum $4_{41<-4}$ Q-branch transition of $\mathrm{NO}_{2}$ at $1613.2 \mathrm{~cm}^{-1}$ with an optical power of $\sim 135 \mathrm{~mW}$. A custom made $22.47 \mathrm{~cm}$ long multipass gas cell (MPGC) was implemented in which the EC-QCL radiation was passed 45 times and provided an effective optical path length of $10.1 \mathrm{~m}$ in order to design a compact sensor system with high detection sensitivity. An air core solenoid surrounding the MPGC was used for generating a magnetic field of 200 Gauss. Performance details of a FRS based sensor and its application for long time continuous $\mathrm{NO}_{2}$ measurements are reported. The long time stability of the sensor system was evaluated. $\mathrm{A} \mathrm{NO}_{2}$ detection sensitivity based on an Allan deviation plot of $\sim 95 \mathrm{ppt}$ was obtained by averaging the associated data for 300 seconds.
\end{abstract}

Key words: nitrogen dioxide detection, mid-infrared external cavity quantum cascade laser, Faraday rotation spectroscopy

\section{Introduction}

Atmospheric pollution has become a serious issue as a result of the recent population growth and industrial development as well as an increased use of fossil fuels. Nitrogen dioxide $\left(\mathrm{NO}_{2}\right)$, like sulfur oxides, carbon dioxide, and carbon monoxide are major primary pollutants in the atmosphere. The major sources of $\mathrm{NO}_{2}$ are mainly from fossil fuel combustion processes, such as the exhaust of motor vehicles, electric power plants and industry. $\mathrm{NO}_{2}$ is also emitted from biomass burning events and via natural processes such as during lightning events and as the result of microbial processes in the soil [1]. 
Nitrogen dioxide also contributes to human health and the environment. In addition, nitrogen dioxide plays a key role in the tropospheric chemistry reactions that produce ground level ozone $\left(\mathrm{O}_{3}\right)$, which is also an important atmospheric pollutant. Furthermore, $\mathrm{NO}_{2}$ contributes to global climate change by interfering, both directly and indirectly, with the earth's radiation budget [2-4]. According to the 2007 Fourth Assessment Report [2], anthropogenic $\mathrm{NOx}\left(\mathrm{NO}\right.$ and $\mathrm{NO}_{2}$ ) emissions have increased drastically since preindustrial times. Therefore, there is an increasing need for the sensitive and selective detection of $\mathrm{NO}_{2}$ in environmental atmospheric monitoring and climate research.

Conventional methods such as chemiluminescence and wet chemical analysis are the most common methods that are widely used for measurements of ambient $\mathrm{NO}_{2}$ concentration levels. However, these methods have certain drawbacks such as that they are slow (minutes-hours) and non selective in discriminating between $\mathrm{NO}$ and $\mathrm{NO}_{2}$, particularly at low concentrations. Infrared tunable diode laser absorption spectroscopy (TDLAS) is an extremely effective tool for the detection and quantification of molecular trace gases, which offers advantages that include fast response, high sensitivity, in situ and non contact measurements [5]. There are several traditional techniques for the detection of trace gases based on laser absorption spectroscopy, such as TDLAS, photoacoustic spectroscopy (PAS), quartz enhanced photoacoustic spectroscopy QEPAS), cavity ring down (CRD) and cavity enhanced spectroscopy (CES), which have been applied to $\mathrm{NO}_{2}$ detection [5-12]. For highly sensitive and selective detection of atmospheric trace gases it is important to select an absorption line with high intensity and minimum interference from other molecules and especially to be free from the influence of water vapor and carbon dioxide.

There are strong absorption lines in the $\mathrm{v}_{3}$ band of $\mathrm{NO}_{2}$, which can achieve high sensitivity by means of TDLAS, but which is limited by interference from $\mathrm{H}_{2} \mathrm{O}$ absorption lines. Therefore, it is difficult to obtain sensitive and selective $\mathrm{NO}_{2}$ detection with TDLAS. However, since $\mathrm{NO}_{2}$ is a paramagnetic molecule there is a potential method for highly selective and sensitive $\mathrm{NO}_{2}$ detection based on Faraday rotation spectroscopy (FRS), which was first reported by G. Litfin et al. in the 1980s with a color-center laser source [13]. FRS is a useful technique for quantification of paramagnetic trace gases with significantly higher sensitivity compared to TDLAS. The three basic noise sources in absorption spectroscopy measurements are source, detector, and quantum noise. Source noise results from amplitude fluctuations of the excitation laser intensity. The high sensitivity of FRS is achieved by significantly reducing source noise [13]. FRS offers the advantage of being free from the influence of non-paramagnetic molecules, such as water and carbon dioxide. This make FRS a suitable technique 
for high selective detection of paramagnetic molecules of atmospheric gases such as $\mathrm{NO}, \mathrm{NO}_{2}, \mathrm{O}_{2}$ and $\mathrm{OH}$ [14-20]. For example, Lewicki et al. reported highly sensitive NO detection at $5.33 \mu \mathrm{m}$ by employing the FRS technique using an external cavity quantum cascade laser (EC-QCL), with a minimum detection sensitivity of $4 \mathrm{ppbv}$ [18]. Zhao et al. reported sensitive $\mathrm{OH}$ detection at $2.8 \mu \mathrm{m}$ using FRS [19]. In 2011 So et al. obtained a minimum detection sensitivity of 30 ppmv for $\mathrm{O}_{2}$ detection by means of the FRS technique [20].

Faraday rotation spectroscopy exploits magnetic circular birefringence (MCB) observed in the vicinity of Zeeman split absorption lines. The transition of paramagnetic molecules (such as $\mathrm{NO}_{2}$ ) will undergo Zeeman splitting and split into $\Delta \mathrm{M}_{\mathrm{J}}=+1$ and $\Delta \mathrm{M}_{\mathrm{J}}=-1$ components, when $\mathrm{NO}_{2}$ is immersed in a longitudinal magnetic field. When linearly polarized light, which can be considered as a superposition of both RHCP and LHCP light, propagates for a distance $\boldsymbol{L}$ through paramagnetic molecules immersed in a longitudinal magnetic field, its plane of polarization will rotate with an angle of $\theta=\Delta n L \pi / \lambda$, as a result of MCB. The concentration of the targeted paramagnetic molecules is proportional to $\Delta n=n_{R}-n_{L}$, the difference between the refractive index for RHCP $\left(n_{R}\right)$ and LHCP $\left(n_{L}\right)$ respectively. A common approach for generating FRS signals is based on an applying an alternating magnetic field, which modulates the Zeeman splitting of the absorption lines. This produces a varying MCB and results in a modulated polarization rotation of the transmitted light. After passing through the multipass gas cell, a second polarizer transforms this polarization rotation into a modulation of the light intensity prior to reaching the detector, which is then demodulated using a phase-sensitive lock-in amplifier. More details of the FRS theory are described in Refs [13,18 -22].

FRS requires that the excitation laser is single-frequency, tunable, operates at room temperature, is mode hop free and that the output wavelength coincides with the targeted molecular absorption line. In this work, an external cavity quantum cascade laser (EC-QCL) tunable between $1600 \mathrm{~cm}^{-1}$ and 1650 $\mathrm{cm}^{-1}$ was used, which allowed targeting the optimum $4_{41} \leftarrow 4_{40}$ Q-branch of the $\mathrm{NO}_{2}$ transition at $1613.25 \mathrm{~cm}^{-1}[14]$.

\section{Sensor design}

The FRS based $\mathrm{NO}_{2}$ sensor platform is schematically shown in Fig. 1. This platform is an improved version of the double pass configured $\mathrm{NO}_{2}$ FRS system that was reported in Ref. [14]. Some original results of the current sensor were also reported in ref. [15]. One major improvement (or change) was using a multipass cell to replace double pass cell in ref. [14], and a detection sensitivity of $250 \mathrm{ppt}$ 
was achieved with time constant of 1s, 4 times was improved than ref. [14]. In addition, laser wave length scanning with a PID controller was used in the current version for continuous, long time field measurement without drift and fluctuations of the reported sensor system.

The optical elements were mounted on an optical breadboard (18 x 24 in. in size), which makes the sensor convenient for deployment in field measurements. The temperature of the EC-QCL was set to operate at $18^{\circ} \mathrm{C}$, which required the use of a water chiller. Experimental investigation showed in Ref. [14] that the $4_{41} \leftarrow 4_{40}$ Q-branch $\mathrm{NO}_{2}$ transition at $1613.25 \mathrm{~cm}^{-1}$ provides a strong FRS signal with clear fingerprint. Therefore, this transition was selected, and the tunable EC-QCL allows targeting this $\mathrm{NO}_{2}$ transition with an optical power of $\sim 135 \mathrm{~mW}$. Fig. 2 depicts the EC-QCL output power in the spectral tuning range, including an optical power drop at $\sim 1635 \mathrm{~cm}^{-1}$, caused by a $\mathrm{H}_{2} \mathrm{O}$ absorption line. Additional components of the $\mathrm{NO}_{2}$ sensor system include a multipass gas cell (MPGC) and a reference cell. The reference cell was filled with a $\sim 1 \% \mathrm{NO}_{2}$ in $\mathrm{N}_{2}$ mixture at a pressure of 25 Torr for control of the EC-QCL frequency. The MPGC was surrounded by a $15 \mathrm{~cm}$ long air core solenoid for generating a magnetic field inside the MCGP. The reference cell was also surrounded by a $10 \mathrm{~cm}$ long solenoid. The two solenoids surrounding the MPGC and the reference cell consisted of a series resonant circuit (SRL) with a $1 \mu \mathrm{F}$ capacitor and the resonant frequency was found to be $f_{m}=1971 \mathrm{~Hz}$. The solenoid current was supplied by a sinusoidal wave from a function generator at a frequency, matched to $f_{m}$ and amplified by a commercial audio amplifier to generate the required AC magnetic field for FRS. Fig. 3 depicts the magnetic field amplitude as a function of the solenoid current, as measured by a gauss meter (FW Bell model 5180).

The MPGC used two gold coated concave mirrors with a diameter of 2 inches and a focal length of $200 \mathrm{~mm}$ and maximum reflectivity of $98.4 \%$ at $\sim 6 \mu \mathrm{m}$. The distance between two mirrors was 22.47 $\mathrm{cm}$, which resulted in an effective optical pathlength of $10.1 \mathrm{~m}$ with 45 laser beam passes. A theoretical analysis of the FRS signal strength versus the pass number of laser beam in the cell can be found in Ref. [16], in which the maximum signal was expected with 63 passes. The photo in Fig. 1 depicts a sample pattern of beam spots on the first mirror. The MPGC was designed in such a way as to tilt the angle of each mirror and to adjust the distance between two MPGC mirrors to achieve different effective optical pathlengths.

The collimated EC-QCL beam was split by a $\mathrm{CaF}_{2}$ wedge into 2 independent optical paths. The main path propagated through the MPGC and with 45 passes. The MPGC was placed between 2 nearly crossed $\mathrm{MgF}_{2}$ Rochon polarizers with an extinction ratio of $<10^{-5}$. The first polarizer was used to 
maintain the incident beam polarized horizontally whereas the second polarizer oriented at an $\sim 90$ crossed position with respect to the first polarizer is used to measure the rotation of the polarization axis caused by the presence of $\mathrm{NO}_{2}$ molecules. The optical power after the first polarizer was measured to be $84 \mathrm{~mW}$ and decreased to $41 \mathrm{~mW}$ after passing 45 times inside the MPGC. The Faraday rotated light passing through a second polarizer and was detected by a mid-infrared thermoelectrically cooled mercury-cadmium-telluride (MCT) photodetector (Vigo, model PDI-2TE-5). The AC magnetic field results in an amplitude modulation of the transmitted light intensity which was detected by a phase sensitive lock-in amplifier at the frequency $f_{m}$. The spectrum of detected FRS signal was recorded by a personal computer connected to a National Instrument data acquisition (NI-DAQ) card. For small rotation angles, the detector signal is directly proportional to the $\mathrm{NO}_{2}$ concentration inside the MPGC.

The second optical path was used as a reference channel for controlling the EC-QCL frequency. Initially the laser beam was transformed into circular polarization by a quarter wave plate. The beam was then directed through the reference cell filled with $1 \% \mathrm{NO}_{2}$ in $\mathrm{N}_{2}$ at a pressure of 25 Torr. The Zeeman modulation signal resulting from magnetic circular dichroism was detected by a thermoelectrically cooled MCT photodetector and demodulated by a lock in amplifier at the second harmonic frequency, $f_{m}$. The laser wavelength was scanned by a sine wave with a frequency of $1 \mathrm{~Hz}$ and 500 points data were acquired for each period. The first peak position of the second harmonic was used to control the laser frequency at the desired position. In case of a frequency shift from the original position a correction signal from the LabView software based PID controller was applied to the EC-QCL piezo element.

\section{Sensor Optimization}

The performance of the FRS based sensor system depends on several factors, such as the amplitude of magnetic field, the pressure of sample gas and the analyzer angle. The FRS signal is proportional to the amplitude of the magnetic field. Fig. 4 shows the FRS signals as a function of amplitude of magnetic field which was acquired with a mixture of $500 \mathrm{ppb} \mathrm{NO}_{2}$ in $\mathrm{N}_{2}$. The use of a magnetic field may affect the FRS signal due to the heating of the two solenoids. A magnetic field amplitude of 200 Gauss was selected, which corresponded to a current of 5 ampere and was compatible for continuous operation (a solenoid current of up to 10 ampere was feasible for short time durations). FRS signals with different pressures were measured for determining the optimum pressure for $\mathrm{NO}_{2}$ detection. In these measurements, the $\mathrm{NO}_{2}$ concentration was $100 \mathrm{ppb}$ for the different pressures. The 
largest FRS signal was obtained at a pressure of 30 Torr, which was selected for $\mathrm{NO}_{2}$ concentration measurements. The performance of a FRS sensor is sensitive to the analyzer angle and must be optimized. Based on the theory introduced in Ref [18], the power transmitted through the analyzer can be expressed as

$$
P=P_{0}\left[\sin ^{2}(\alpha)+\xi\right]
$$

and the FRS signal demodulated by lock-in amplifier can be expressed as

$$
S=\kappa \Delta \Phi P_{0} 2 \alpha=a \alpha P_{0}
$$

where $P_{0}$ is the intensity of light incident at the analyzer, $\alpha$ is the analyzer angle with respect to its crossed position, $\xi$ is the polarizer extinction ratio, $\kappa$ is the proportionality constant including the detector response, $\Delta \Phi$ is the rotation angle of the polarization of the incident light as a result of Faraday effect and $a=2 \kappa \Delta \Phi$. For a small $\alpha$, the SNR can be expressed as

$$
S N R=\frac{a \alpha P_{0}}{\sqrt{b^{2}+P_{0} c^{2}\left(\alpha^{2}+\xi\right)+P_{0}^{2} d^{2}\left(\alpha^{2}+\xi\right)^{2}}}
$$

where $b$ is detector noise equivalent power (NEP), $c \sqrt{P_{0}\left(\alpha^{2}+\xi\right)}$ is the tranmitted quantum nose, $\mathrm{c}$ is a coefficient related to detector responsivity, and $P_{0} d\left(\alpha^{2}+\xi\right)$ is the transmitted source noise (d being the proportionality coefficient specific for the used laser source). The optimum angle $\alpha_{o p t}$ that guarantees a maximum signal-to-noise can be determined by setting the derivative of the SNR with respect to $\alpha$ equal to zero, and solving for $\alpha$ :

$$
\alpha_{o p t}=\left[\left(\frac{b}{P_{0} d}\right)^{2}+\left(\frac{c \sqrt{P_{0} \xi}}{P_{0} d}\right)^{2}+\xi^{2}\right]^{1 / 4}
$$

For a small $\xi$ or a small laser power $P_{0}$, the optimum system performance can be achieved when the laser noise equals the detector noise, namely:

$$
\alpha_{o p t}=\sqrt{\frac{b}{P_{0} d}}
$$

Details of the analysis of signal-to-noise ratio (SNR) related to the angle of analyzer are reported in references [18-20]. In the present work, a series of measurements were performed for the same gas mixture at different analyzer angles to determine the optimum $\alpha_{o p t}$ for the optimum SNR value of the FRS system. Fig. 5 shows the results of FRS signal and SNR for different analyzer angles $\alpha$, which were measured with a $100 \mathrm{ppb} \mathrm{NO}_{2}$ in $\mathrm{N}_{2}$ concentration at an optimum pressure of 30 Torr. The FRS 
signal decreases when the analyzer angle $\alpha$ is $>5$ degrees as the EC-QCL beam will saturate the MCT detector. The maximum SNR was obtained at an angle, $\alpha=2$ degree.

\section{Sensor performance evaluation}

A performance evaluation based on the linearity response after optimization of the FRS based $\mathrm{NO}_{2}$ sensor system with respect to $\mathrm{NO}_{2}$ concentration levels was performed. A calibrated standard gas mixture consisting of 2 ppm $\mathrm{NO}_{2}$ : $\mathrm{N}_{2}$ was employed and diluted with pure $\mathrm{N}_{2}$ using two MKS flow meters. One flow meter with a range from 0 to $200 \mathrm{ccm}$ was used to control the flow rate of the calibrated standard gas. The other flow meter with a flow range from 0 to $1,000 \mathrm{ccm}$ was used to control the flow rate of pure $\mathrm{N}_{2}$. Fig. 6 depicts the results of the FRS signal as a function of the $\mathrm{NO}_{2}$ concentration.

Fig. 7 (a) shows a FRS spectrum of $\mathrm{NO}_{2}$ in ambient air that was obtained by slowly scanning the EC-QCL wavelength at a scanning rate of $2 \mathrm{mHz}$, from which a noise level of $1 \sigma=0.24 \mu \mathrm{V}$ was determined. The corresponding SNR was found to be 13 and the $\mathrm{NO}_{2}$ concentration shown in Fig. 7 (a) was determined to be $3.3 \mathrm{ppb}$ based on the linearity plot shown in Fig. 6. With these parameters, a minimum detection limit of $250 \mathrm{ppt}$ was obtained for a 1 s time constant. However, a longer time constant can be implemented by locking of the laser frequency to the center of $\mathrm{NO}_{2}$ absorption line [14] in order to further improve detection sensitivity.

In the following measurements, fast scanning of the laser wavelength and data averaging with different time schemes was employed. In this case the EC-QCL laser wavelength is scanned across the selected $4_{41}<-4_{40} \mathrm{NO}_{2}$ transition with a frequency of $1 \mathrm{~Hz}$ and a time constant of $10 \mathrm{~ms}$. The peak position of the $2 \mathrm{f}$ reference signal was continuously monitored and a correction signal from a LabView software based PID controller was applied to the EC-QCL piezo element. In this manner, data with an averaging time of 10 second, 100 second and 300 second, was acquired. As an example, Fig. 7 (b) depicts the FRS spectrum of $50 \mathrm{ppb} \mathrm{NO}_{2}$ acquired without averaging, averaging of 10 second, 100 second and 300 second, respectively (note: base line offset were performed for each spectrum). The noise level decreased significantly by using this approach. The minimum detection limit for 1 second, averaged in 10, 100 and 300 seconds were found to be $2.5 \mathrm{ppb}, 1.4 \mathrm{ppb}, 700 \mathrm{ppt}$ and $240 \mathrm{ppt}$ respectively. Hence the FRS based $\mathrm{NO}_{2}$ sensor system can detect $\mathrm{NO}_{2}$ with a MDL of 2.5 ppb in 1 second and a MDL as low as 240 ppt by averaging for 300 seconds. The FRS sensor noise level was also evaluated based on an Allan-Werle deviation analysis shown in Fig 8 [23]. Figure 8 shows that the 
FRS sensor system allows averaging times of up to 600 seconds. Hence, the minimum detection limits can be of the order of $500 \mathrm{ppt}, 150 \mathrm{ppt}$ and $95 \mathrm{ppt}$ by averaging the data for 10 seconds, 100 seconds and 300 seconds, respectively. The response time of the sensor is not a critical parameter in the case of environmental monitoring, especially, when the concentration of the targeted molecules is low and a long data averaging time is beneficial for the improved measurement accuracy and precision.

\section{Application of a FRS based sensor system in atmospheric $\mathrm{NO}_{2}$ concentration measurements}

Validation measurements of the developed FRS based $\mathrm{NO}_{2}$ sensor for field applications were performed by continuously monitoring of atmospheric $\mathrm{NO}_{2}$ at two sites located at Rice University and University of Houston, respectively. The FRS based sensor was first operated in the Rice Laser Science Group laboratory (Space Science \& Technology Bldg. Suite 104) followed with air from outside the laboratory collected via a sampling tube from outside the laboratory. The sampled outside air continuously flowed into the MPGC by pumping continuously. The continuous measurements lasted >48 hours. Several distinct peaks, of up to $600 \mathrm{ppb} \mathrm{NO}_{2}$ in the air, were observed during day time, as shown in Fig. 9. During the measurement period, a mobile crane was operated outside SST 104, which resulted in sharp $\mathrm{NO}_{2}$ absorption peaks from the exhaust of the crane engines. Hence the reported FRS based $\mathrm{NO}_{2}$ sensor system can be useful in monitoring of $\mathrm{NO}_{2}$ emissions from motor vehicles.

The University of Houston field measurements were carried out on the top of the Moody Tower, a $\sim 60 \mathrm{~m}$ high building located $\sim 5$ miles from the Rice University site. At this same site, a similar campaign for measuring atmospheric ammonia $\left(\mathrm{NH}_{3}\right)$ with a photoacoustic spectroscopy technique was carried out by a RLSG team in the 2010 [24]. The FRS based sensor performed continuously unattended, real-time concentration measurements of atmospheric $\mathrm{NO}_{2}$. The sensor was capable of remote access which enabled real-time monitoring of the sensor performance from anywhere via internet. This function was successfully demonstrated by controlling the $\mathrm{NO}_{2}$ sensor located in Houston, TX from Hefei, China, 10,000 miles from its location in the USA. The FRS based sensor was operated for more than one month continuously. Fig. 10(a) shows the diurnal plot of $\mathrm{NO}_{2}$ acquired during the campaign from September 12, 2011 to October 28, 2011. The black dots in the Fig. 10(a) show the mean values of the $\mathrm{NO}_{2}$ concentration. A diurnal plot of ozone $\left(\mathrm{O}_{3}\right)$ is shown in Fig. 10(c) for convenient analysis. Fig. 10(b) shows the total, weekday and weekend diurnal plots of the $\mathrm{NO}_{2}$ mean value obtained during the campaign, respectively. Fig. 10(b) shows that the peak mean values of the 
$\mathrm{NO}_{2}$ concentration occurred at 8 am clock (2 pm clock GMT) with values of $22.45 \mathrm{ppb}$ (total), $23.6 \mathrm{ppb}$ (weekday) and $19.35 \mathrm{ppb}$ (weekend), respectively. The $\mathrm{NO}_{2}$ absorption peak mean values at 8 am (2 pm clock GMT) on weekdays are larger than the values measured on weekends. This demonstrates that $\mathrm{NO}_{2}$ from traffic emissions exhaust contributes to the high $\mathrm{NO}_{2}$ concentration levels in the morning hours. Furthermore, the high $\mathrm{NO}_{2}$ concentration values in the morning are mainly due from the atmospheric chemistry reaction in the evening [1]:

$\mathrm{NO}+\mathrm{O}_{3} \rightarrow \mathrm{NO}_{2}+\mathrm{O}$

$\mathrm{O}+\mathrm{NO}+\mathrm{M} \rightarrow \mathrm{NO}_{2}+\mathrm{M}$

where $\mathrm{M}$ represents $\mathrm{N}_{2}$ or $\mathrm{O}_{2}$ or a third molecule that absorbs the excess vibrational energy and thereby stabilizes the $\mathrm{NO}_{2}$ molecule. One of the key atmospheric chemistry reaction is $\mathrm{NO}_{2}$ photolysis. During evening hours, the rapid decrease of the $\mathrm{NO}_{2}$ photolysis rate and the abundance of atomic oxygen lead to the conversion of $\mathrm{NO}$ into $\mathrm{NO}_{2}$. Thus the total number of $\mathrm{NO}_{2}$ molecules can increase substantially in morning hours and reach their highest values at $\sim 8$ am (2 pm clock GMT). After 8 am (2 pm clock GMT), with the increase of sunlight intensity, $\mathrm{NO}_{2}$ photolysis at wavelengths of $<424 \mathrm{~nm}$ occurs as follows [1]

$\mathrm{NO}_{2}+h v \rightarrow \mathrm{NO}+\mathrm{O}$

$\mathrm{O}+\mathrm{O}_{2}+\mathrm{M} \rightarrow \mathrm{O}_{3}+\mathrm{M}$

Hence, the $\mathrm{NO}_{2}$ concentration decreases dramatically after 8 am (2 pm clock GMT) and the concentration of $\mathrm{O}_{3}$ is increased correspondingly, as shown in Fig. 10(a) and Fig. 10(c). The $\mathrm{O}_{3}$ sensor is located at the same location as the FRS sensor and the data of $\mathrm{O}_{3}$ concentration is obtained from the TCEQ website [25].

\section{Conclusions}

A compact, sensitive $\mathrm{NO}_{2}$ sensor based on FRS was developed. The FRS technique offers advantages such as high sensitivity, high selectivity and free from the influence of diamagnetic species. The sensitivity of the present developed FRS based $\mathrm{NO}_{2}$ sensor was enhanced by using a compact MPGC with a $10.1 \mathrm{~m}$ effective optical path length. The performance of the FRS based $\mathrm{NO}_{2}$ sensor was optimized with respect to pressure, amplitude of the applied magnetic field and the angle of the analyzer polarizer. The sensitivity of the FRS based $\mathrm{NO}_{2}$ sensor was found to be 2.5 ppb for $\mathrm{NO}_{2}$ detection with a $10 \mathrm{~ms}$ time constant. The sensitivity can be further improved to $<100 \mathrm{ppt}$ by averaging for 300 s. Long term, continuous, fully automated field measurements of atmospheric $\mathrm{NO}_{2}$ using a FRS 
based $\mathrm{NO}_{2}$ sensor, were performed. Further improvement of the $\mathrm{NO}_{2}$ sensor system $\mathrm{SNR}$ value can be achieved by using better quality polarizers, a more sensitive photodetector, and reducing electronic system noise. The FRS based $\mathrm{NO}_{2}$ sensor developed to date can be used to measure $\mathrm{NO}_{2}$ concentration levels relevant to environmental monitoring, atmosphere chemistry research as well as exhaust analysis of motor vehicles. The FRS based sensor has an application in atmospheric chemistry research as it offers much lower atmospheric background interference than traditional laser absorption spectroscopy.

Acknowledgments: The authors gratefully acknowledge the financial support from the National Natural Science Foundation of China (Nos. 41205120, 41475023), and A US National Science Foundation (NSF) ERC MIRTHE award, a NSF-ANR award NexCILAS, as well as grant C-0586 from the Robert Welch Foundation.

\section{References}

[1] J. H. Seinfeld and S. N. Pandis, Atmospheric Chemistry and Physics: From Air Pollution to Climate Change, John Wiley \& Sons, New York, 1998.

[2] S. Solomon, D. Qin, M. Manning, Z. Chen, M. Marquis, K. B. Averyt, M. Tignor, and H. L. Miller, IPCC: Climate Change 2007: The Physical Science Basis. Contribution of Working Group I to the Fourth Assessment Report of the Intergovernmental Panel on Climate Change, Cambridge University Press, Cambridge, United Kingdom and New York, NY, USA, 2007.

[3] S. Solomon, W. Portmann, R. W. Sanders, J. S. Daniel, W. Madsen, B. Bartram, and E. G. Dutton, On the role of nitrogen dioxide in the absorption of solar radiation, J. Geophys. Res., 104, 12047-12058, 1999.

[4] A. P. Vasilkov, J. Joiner, L. Oreopoulos, J. F. Gleason, P. Veefkind, E. Bucsela, E. A. Celarier, R. J. D. Spurr, and S. Platnick, Impact of tropospheric nitrogen dioxide on the regional radiation budget, Atmos. Chem. Phys., 9, 6389-6400, 2009.

[5] R. F. Curl and F. K. Tittel, Tunable infrared laser spectroscopy, Annu. Rep. Prog. Chem., Sect. C, 98, 219-272, 2002.

[6] K. Liu, X. Guo, H. Yi, W. Chen, W. Zhang, and X. Gao, Off-beam quartz-enhanced photoacoustic spectroscopy, Opt. Lett. 34, 1594 ,200).

[7] W. Chen, A.A. Kosterev, F.K. Tittel, X. Gao, W. Zhao, $\mathrm{H}_{2} \mathrm{~S}$ trace concentration measurements using off-axis integrated cavity output spectroscopy in the near-infrared, Appl. Phys. B 90, $311-315,2008$ 
[8] A. Karpf and G. N. Rao, Absorption and wavelength modulation spectroscopy of $\mathrm{NO}_{2}$ using a tunable, external cavity continuous wave quantum cascade laser, Applied Optics, 48, 408-413, 2009

[9] M. Pushkarsky, A. Tsekoun, I. G. Dunayevskiy, R. Go, and C. K. N. Patel, Sub-parts-per-billion level detection of $\mathrm{NO}_{2}$ using room-temperature quantum cascade lasers, PNAS, 103, 10846-10849, 2006

[10]H. Yi, K. Liu, W. Chen, T. Tan, L. Wang, and X. Gao, Application of a broadband blue laser diode to trace $\mathrm{NO}_{2}$ detection using off-beam quartz-enhanced photoacoustic spectroscopy, Opt. Lett.36, $481,2011$.

[11] M. Bitter, S. M. Ball, I. M. Povey, and R. L. Jones, A broadband cavity ringdown spectrometer for in-situ measurements of atmospheric trace gases, Atmos. Chem. Phys., 5, 2547-2560, 2005.

[12]J. M. Langridge, S. M. Ball and R. L. Jones, A compact broadband cavity enhanced absorption spectrometer for detection of atmospheric $\mathrm{NO}_{2}$ using light emitting diodes, Analyst 131, 916-922, 2006

[13]G. Litfin, C. R. Pollock, R. F. Curl and F. K. Tittel, Sensitivity enhancement of laser absorption spectroscopy by magnetic rotation effect, J. Chern. Phys., 72, 6602-6605, 1980.

[14]C. A. Zaugg, R. Lewicki, T. Day, R. F. Curl, F. K. Tittel,Faraday rotation spectroscopy of nitrogen dioxide based on a widely tunable external cavity quantum cascade laser, Proc. of SPIE, 7945, 794500, 2011

[15]Rafał Lewicki, Kun Liua, Timothy Day, and Frank K. Tittel, Sub-ppb detection of nitrogen dioxide with an external cavity quantum cascade laser, Proc. of SPIE Vol. 8268 82682H, 2012.

[16]Y. Wang, M. Nikodem, B. E. Brumfield, and G. Wysocki, Compact Multi-pass Cell based Faraday Rotation Spectrometer for Nitric Oxide detection, in Conference on Lasers and Electro-Optics, CW3B, 2012.

[17]G. Wysocki, R. Lewicki, X. Huang, R. F. Curl, and F. K. Tittel, Continuous Monitoring of Nitric Oxide at $5.33 \mu \mathrm{m}$ with an EC-QCL based Faraday Rotation Spectrometer: Laboratory and Field System Performance, Proc. of SPIE, 7222, 72220M, 2009.

[18]R. Lewicki, J. H. Doty III, R. F. Curl, F. K. Tittel, and G. Wysocki, Ultrasensitive detection of nitric oxide at $5.33 \mu \mathrm{m}$ by using external cavity quantum cascade laser-based Faraday rotation spectroscopy, PNAS, 106, 12587-12592, 2009.

[19] W. Zhao, G. Wysocki, W. Chen, E. Fertein, D. L. Coq, D. Petitprez, and W. Zhang, Sensitive and 
selective detection of $\mathrm{OH}$ radicals using Faraday rotation spectroscopy at $2.8 \mu \mathrm{m}$, Opt. Express , 19, 2493-2501, 2011.

[20]S.G. So - E. Jeng · G. Wysocki, VCSEL based Faraday rotation spectroscopy with a modulated and static magnetic field for trace molecular oxygen detection, Appl Phys B (2011) 102: 279-291

[21] H. Ganser, W. Urban and J. M. Brown, The sensitive detection of NO by Faraday modulation spectroscopy with a quantum cascade laser, MOL. Phys. 101, 545-550, 2003

[22] C. R. Mahon and C. Chackerian, Zeeman Tuning Rates for Q-Branch Transitions in the $v_{3}$ Band of $\mathrm{NO}_{2}$, J. M. Spectros 189, 276-282, 1998.

[23] P. Werle, R. R. Mücke, F. Slemr, The Limits of Signal Averaging in Atmospheric Trace-Gas Monitoring by Tunable Diode-Laser Absorption Spectroscopy (TDLAS), Appl. Phys. B, 57,131-139 (1993)

[24] L. Gong, R. Lewicki, R. J. Griffin, J. H. Flynn, B. L. Lefer, and F. K. Tittel, Atmospheric ammonia measurements in Houston, TX using an external-cavity quantum cascade laser-based sensor, Atmos. Chem. Phys., 11, 9721-9733, 2011

[25]http:/www.tceq.texas.gov 


\section{Figure captions}

Fig. 1 Schematic of the FRS based $\mathrm{NO}_{2}$ sensor. The photo shows the distribution of beam spots produced by a red diode alignment laser incident on one of the MPGC mirrors.

Fig. 2 EC-QCL output power as a function of its spectral tuning range.

Fig. 3 Magnetic field amplitude as function of solenoid current.

Fig. 4 FRS signal as a function of magnetic field amplitude. The $\mathrm{NO}_{2}$ concentration used in the FRS signal measurement was 500 ppbv.

Fig. 5 FRS signal and SNR as a function of the analyzer angle, $\alpha$.

Fig. 6 Linear dependence of the FRS signal as a function of the $\mathrm{NO}_{2}$ concentration.

Fig. 7 FRS spectrum of $\mathrm{NO}_{2}$ acquired in ambient air (a), and (b) FRS spectrum of $\mathrm{NO}_{2}$ acquired from a 50 ppb $\mathrm{NO}_{2}$ in $\mathrm{N}_{2}$ mixture with different averaging times. Red line in Fig (a) was fitted by using $50 \mathrm{ppb} \mathrm{NO} \mathrm{N}_{2}$ signal as reference.

Fig. 8 Allan deviation plot of the FRS based $\mathrm{NO}_{2}$ sensor system (bottom) and long-time, continuous measurements of $50 \mathrm{ppb} \mathrm{NO}$ (top). Red line in the top represent signal averaged in 1 minute.

Fig. 9 Episode of high concentration of $\mathrm{NO}_{2}$ observed during measurements

Fig. 10 Diurnal profiles of $\mathrm{NO}_{2}$ (a), (b) mean values of $\mathrm{NO}_{2}$ concentration levels during a field measurement campaign in Houston, TX from September 12, 2011 to October 28, 2011, and (c) $\mathrm{O}_{3}$ concentrations obtained during the measurement campaign. The bottom whisker, box bottom, line inside the box, box top, and top whisker represent the 5th, 25th, 50th, 75th, and 95th percentiles of the data, and the dot inside box represent mean values in fig 10 (a) and (c), respectively. 


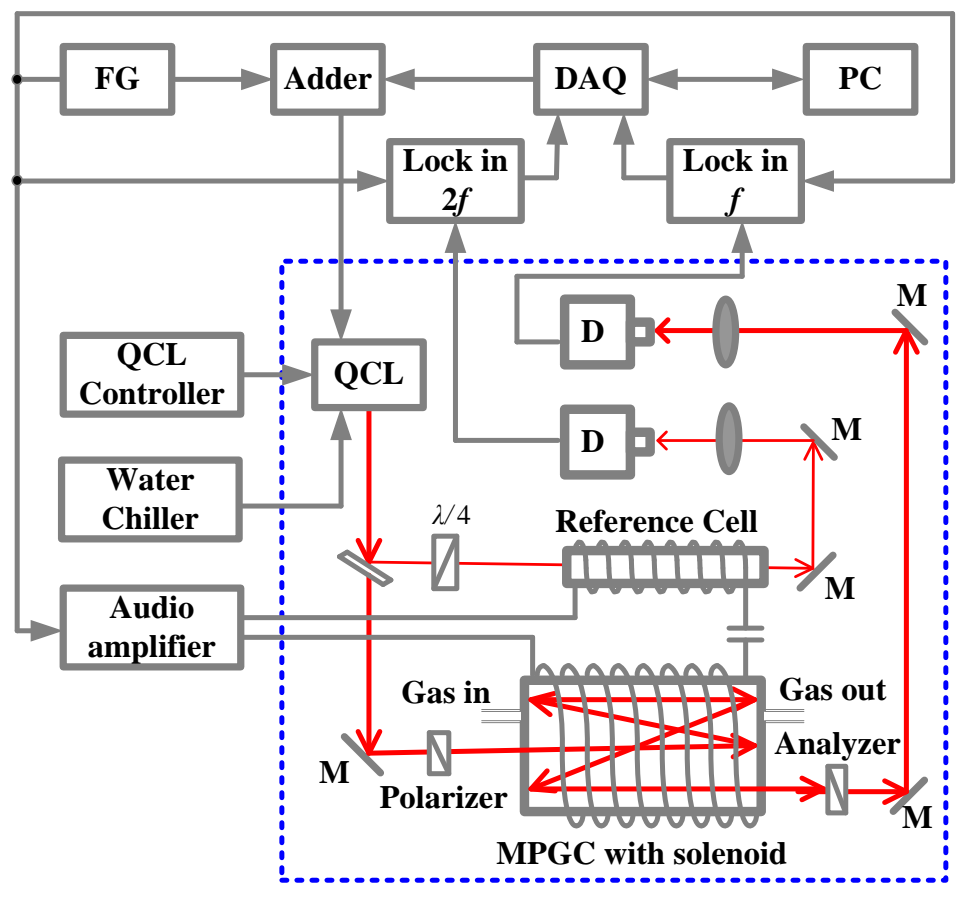

$24 \times 18$ inches

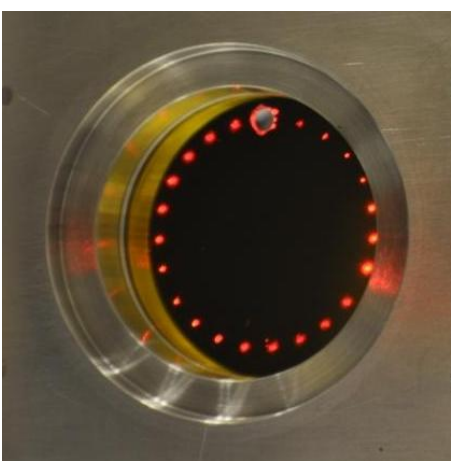

Red diode alignment laser beam spot pattern on one of the two MPGC mirrors

Figure 1 


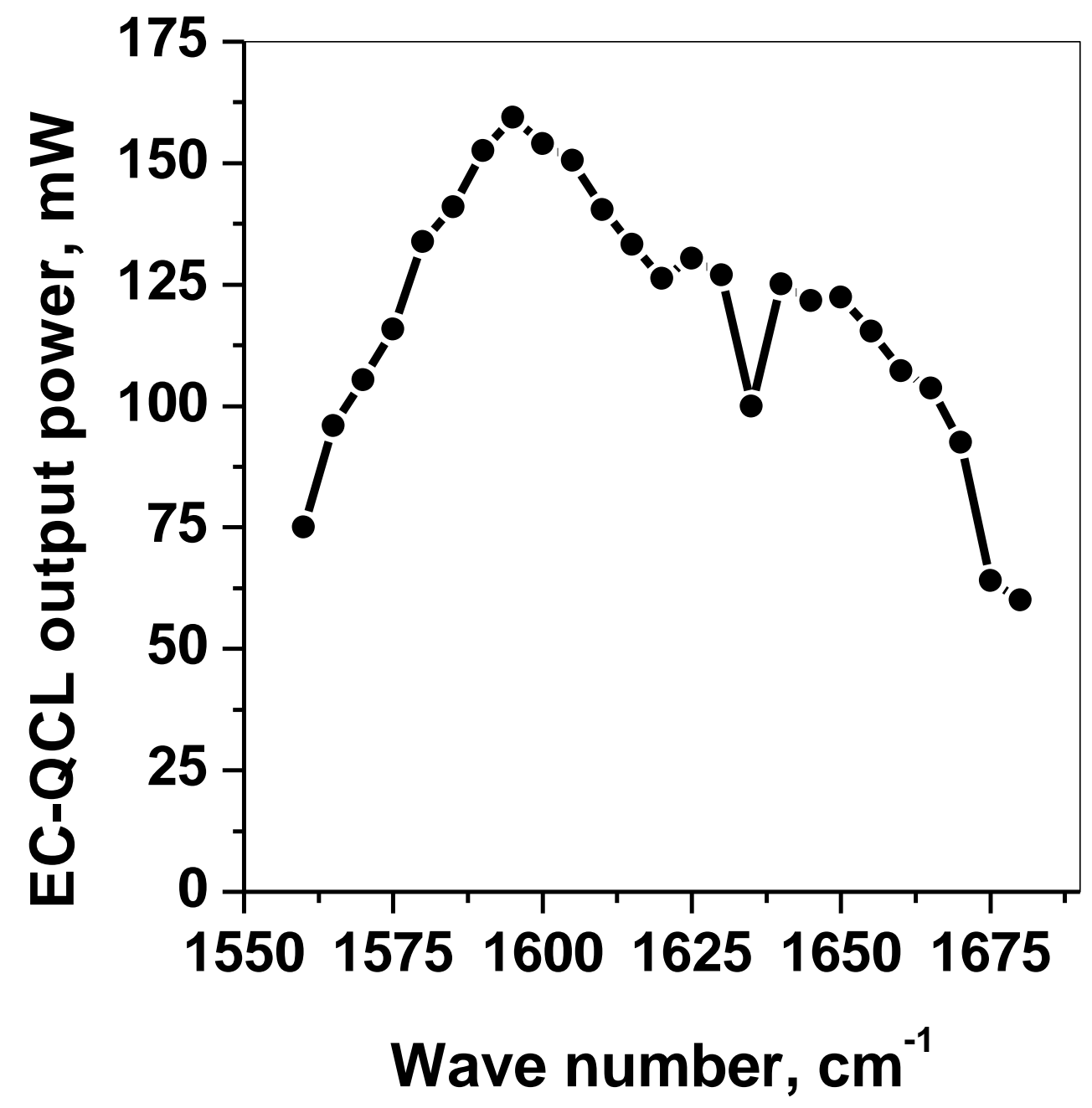

Figure 2 


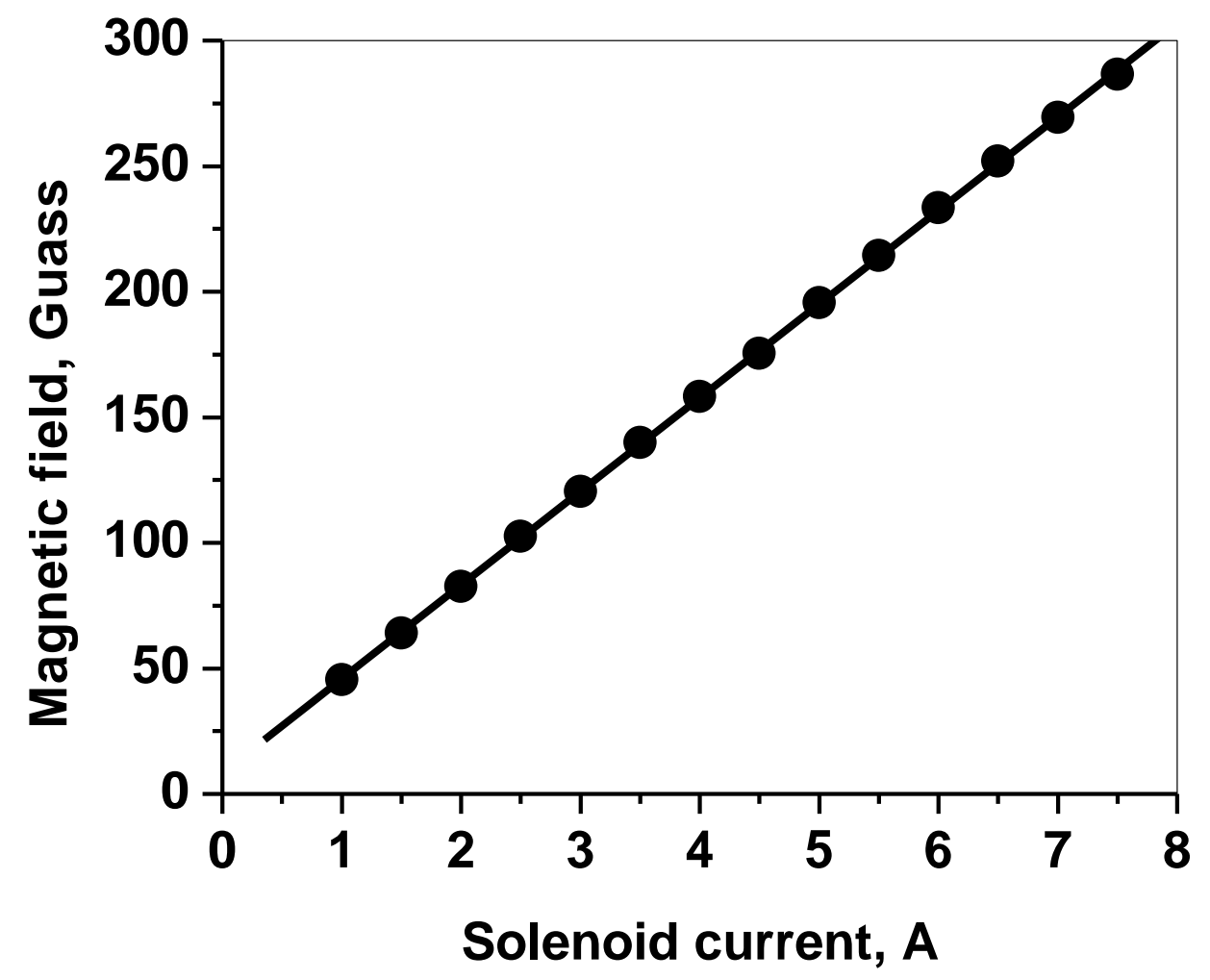

Figure 3 


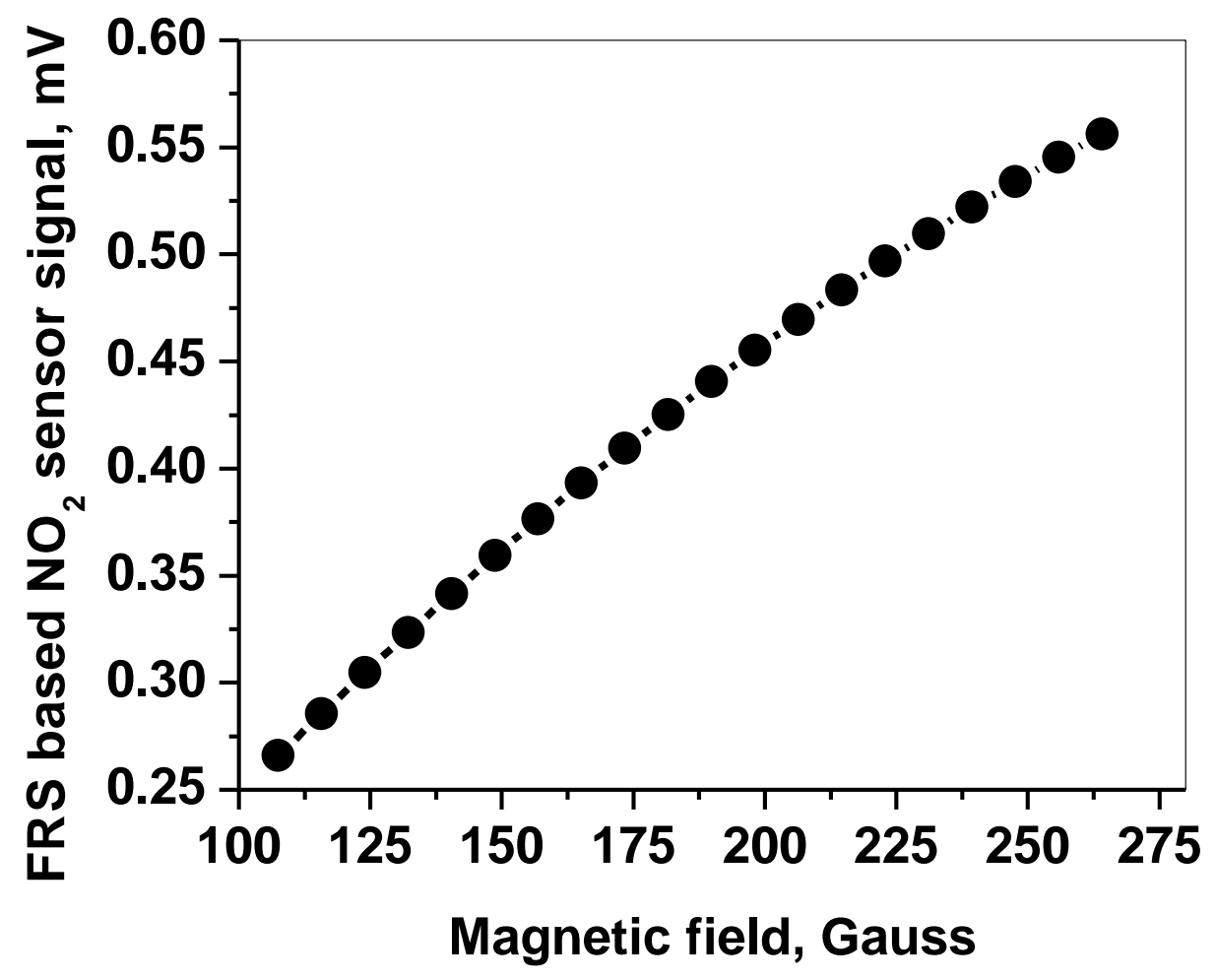

Figure 4 


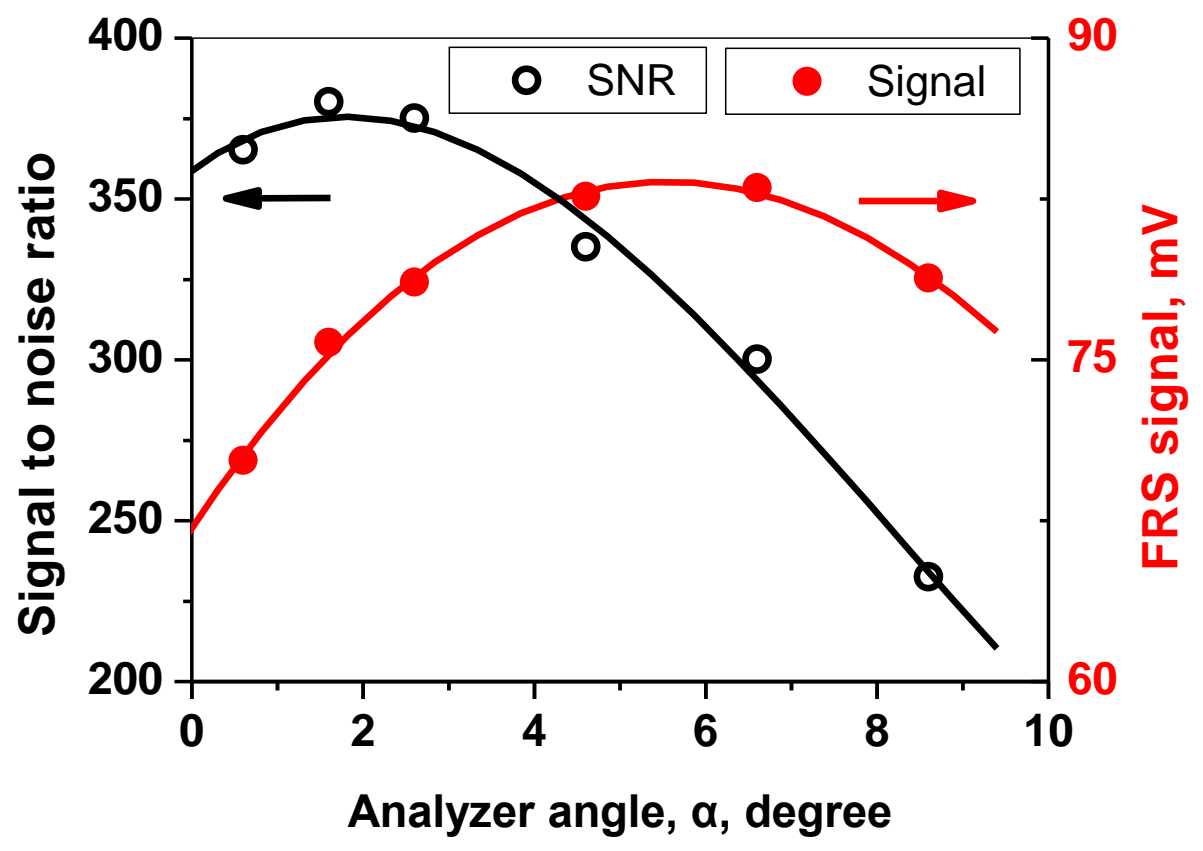

Figure 5 


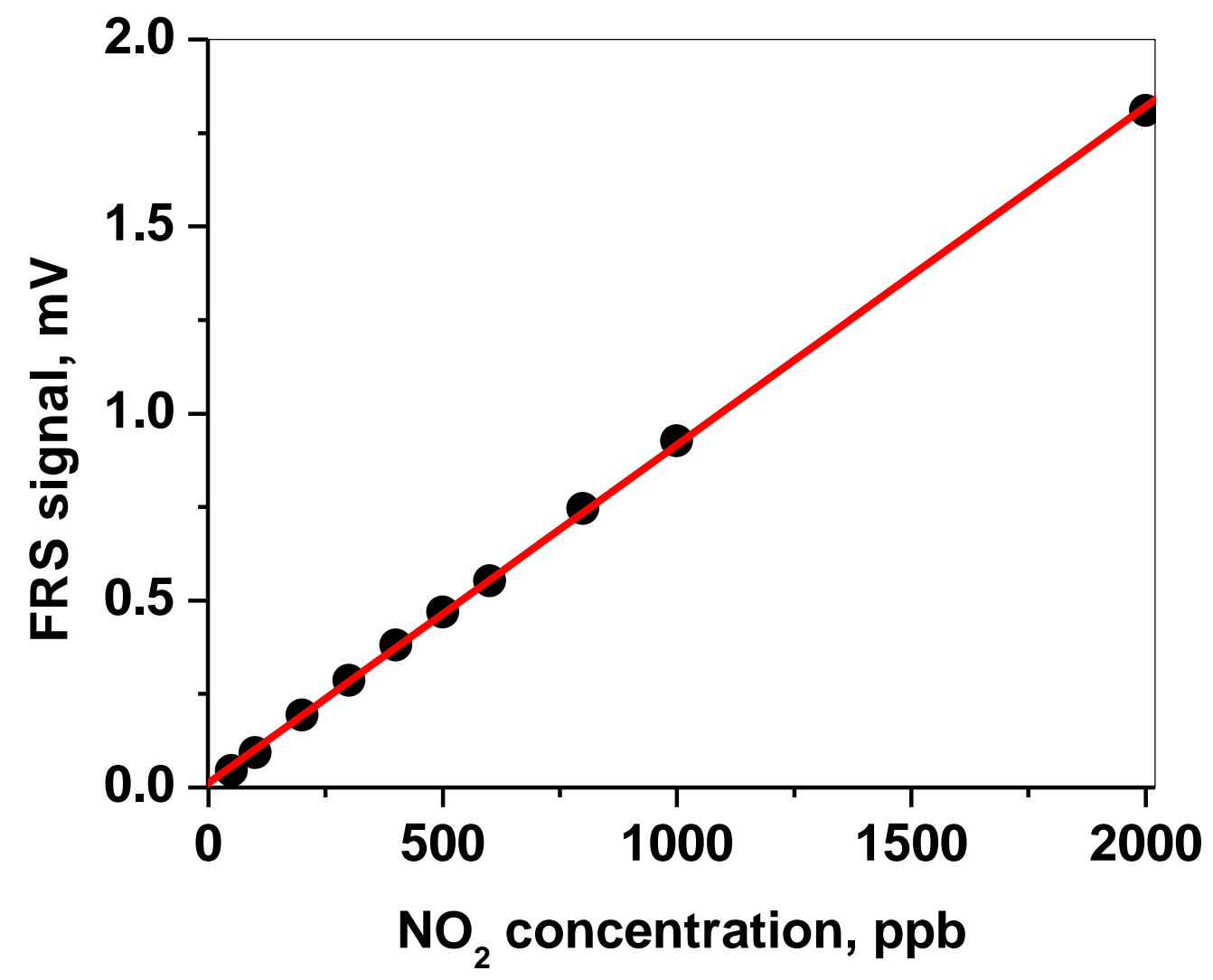

Figure 6 

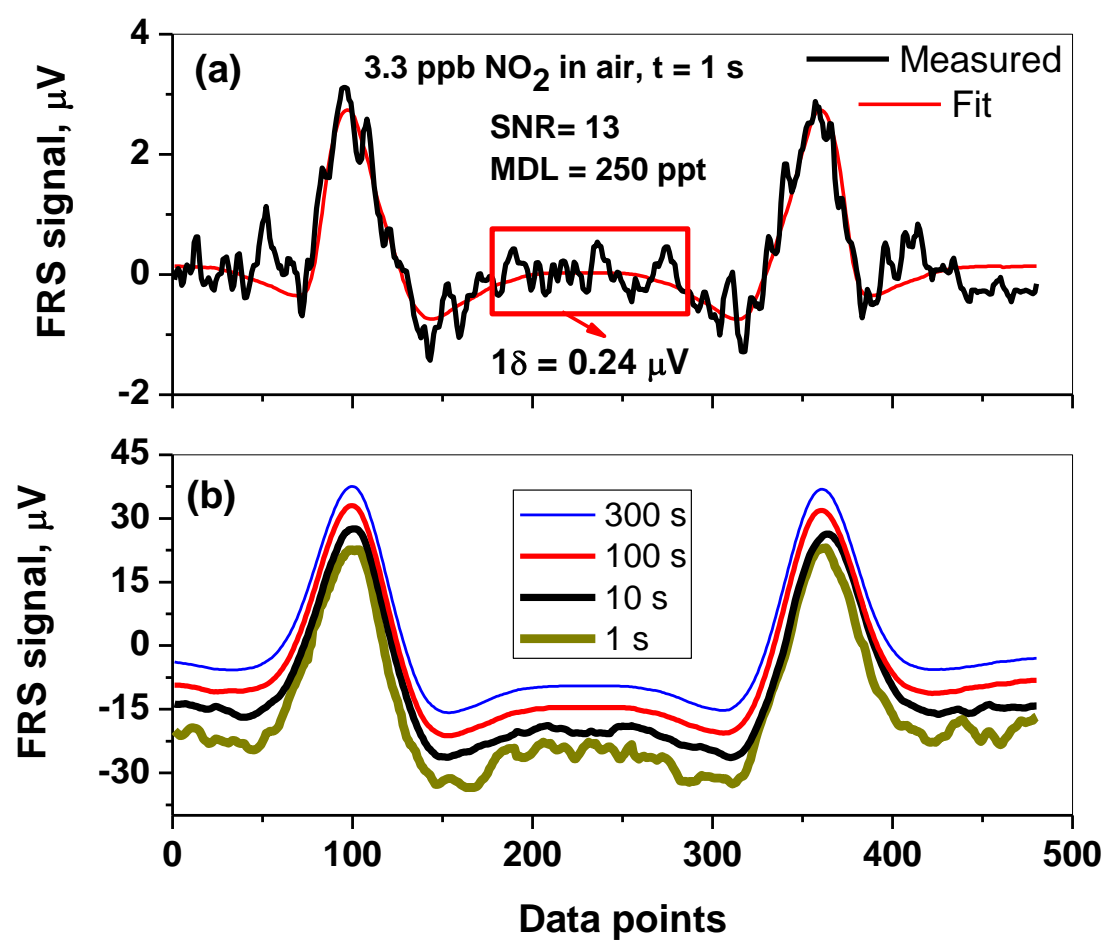

Figure 7 

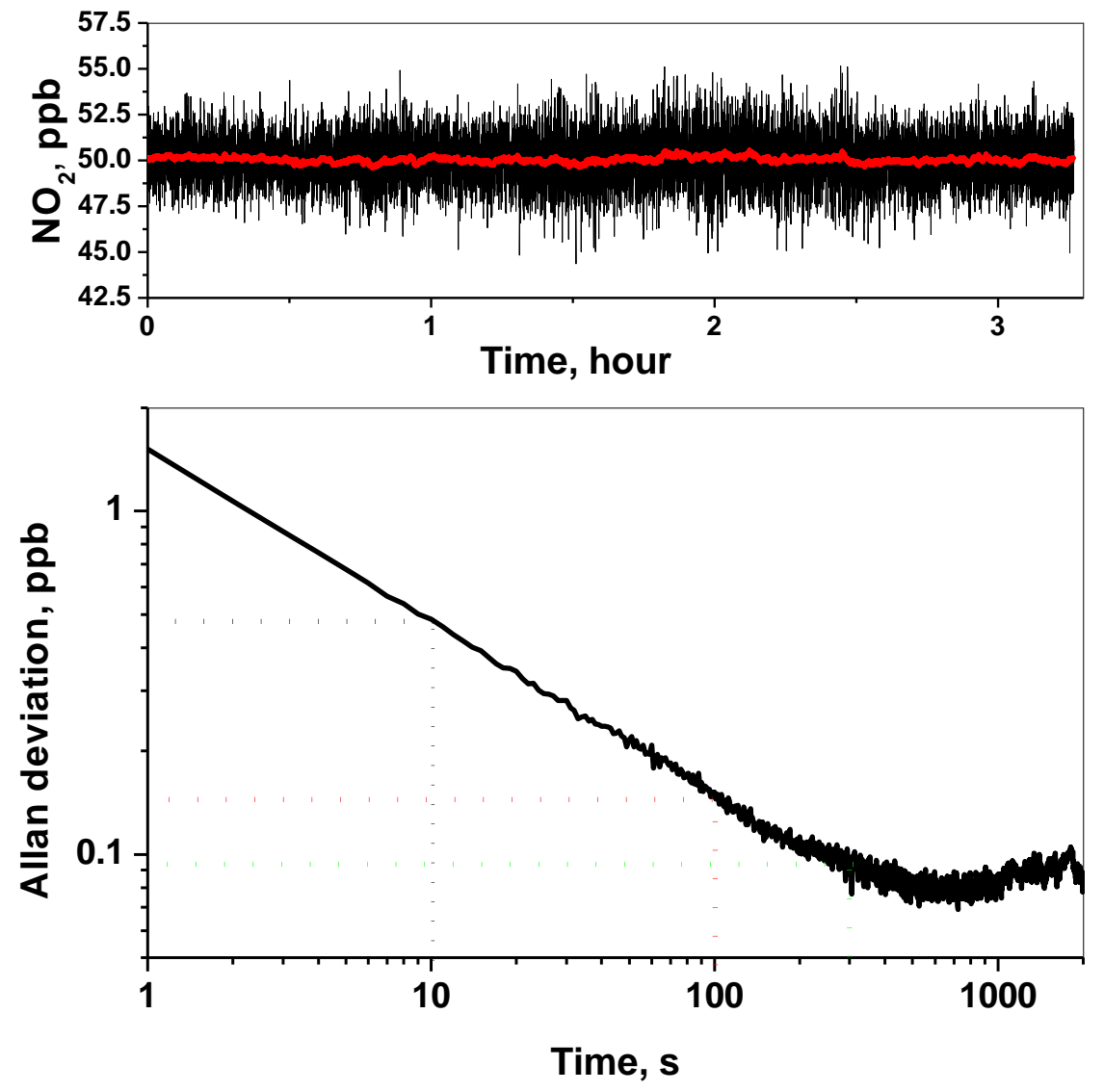

Figure 8 

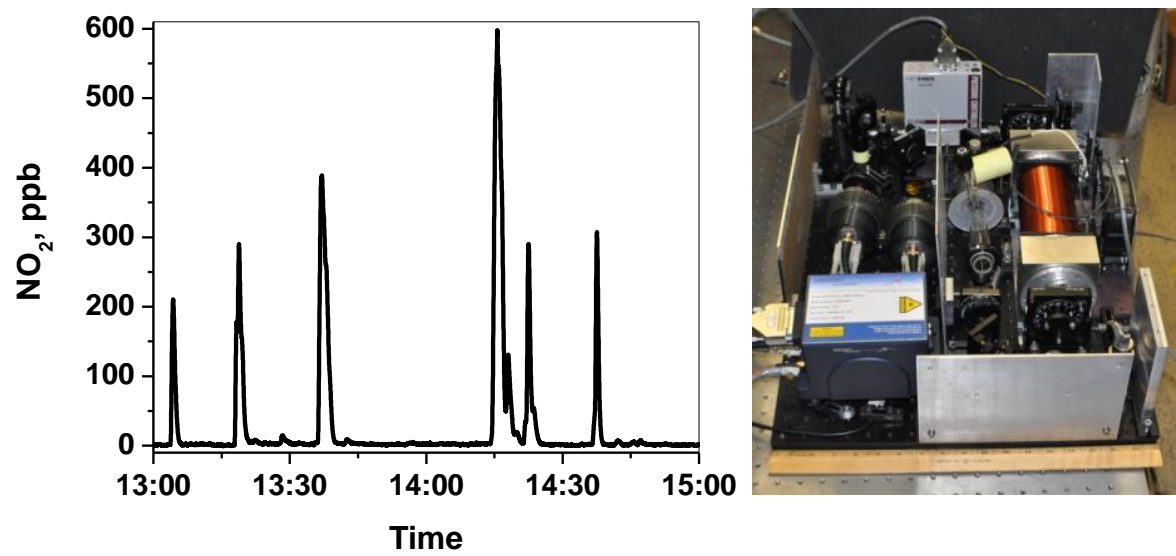

Figure 9 


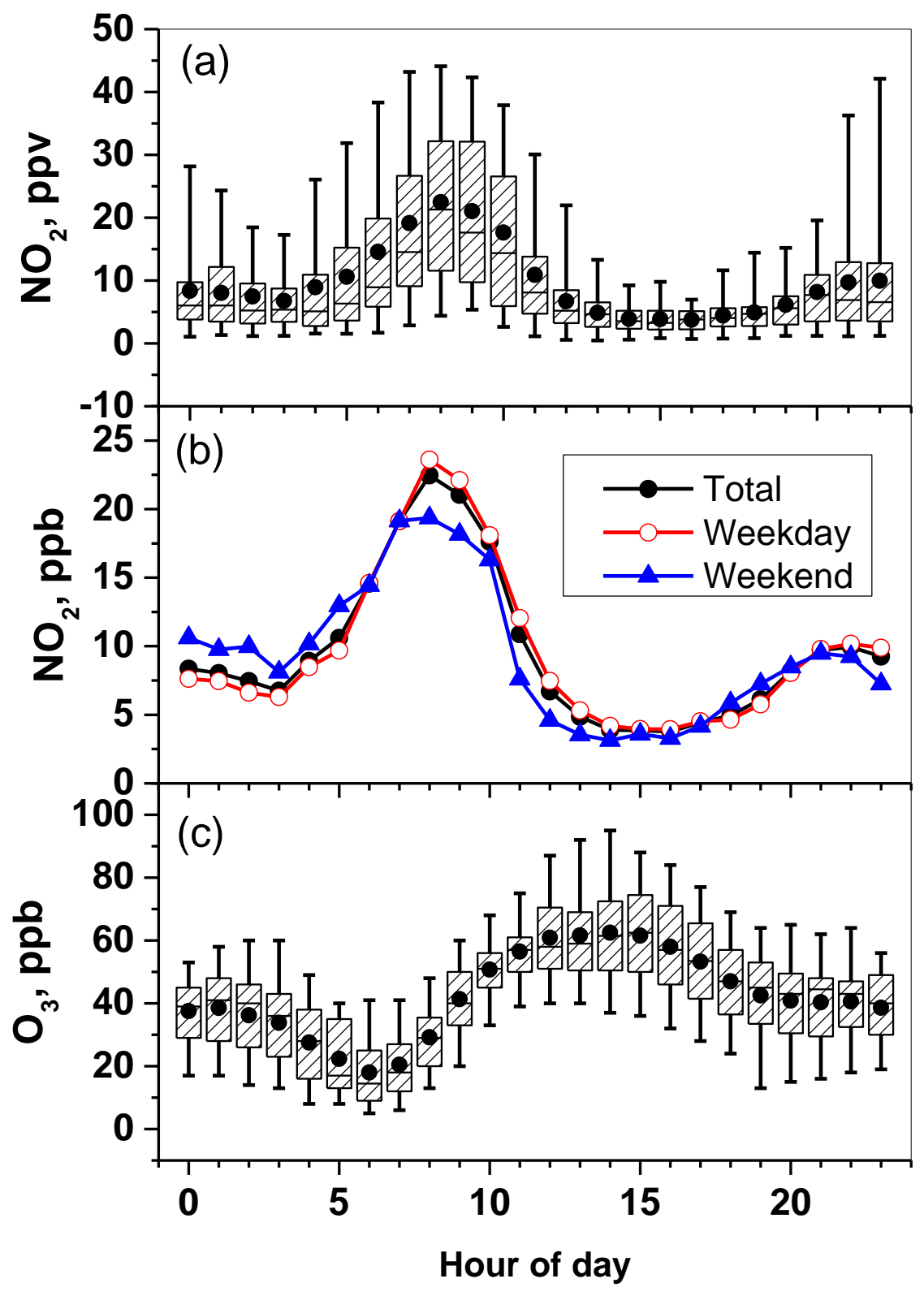

Figure 10 